\title{
Deep-Submicron Structures in YBCO: Fabrication and Measurements
}

\author{
A.J.M. van der Harg, E. van der Drift and P. Hadley \\ DIMES, Feldmannweg 17, NL-2628CT Delft, The Netherlands
}

\begin{abstract}
We present a fabrication method that consistently produces superconducting structures with lateral dimensions down to $100 \mathrm{~nm}$. The etching is done in a Distributed Electron Cyclotron (ECR-) etcher using a plasma of argon and oxygen. The sample is water cooled. Degradation of the etched structures is very limited. We discuss electrical measurements on long, narrow lines and constrictions.
\end{abstract}

\section{INTRODUCTION}

The availability of good quality thin films of $\mathrm{YBa}_{2} \mathrm{Cu}_{3} \mathrm{O}_{7}$ (YBCO) and other high $\mathrm{T}_{c}$ materials has prompted extensive research into ways of patterning such films into useful devices. It is almost impossibe to make devices with lateral dimensions on the scale of the coherence length in YBCO, since it is extremely small $(3-5 \mathrm{~nm})$. The scale of the perpendicular penetration depth $(\sim 400 \mathrm{~nm})$ however, is much more easily accessible. Devices in this regime should exhibit all kinds of effects related to vortex movement and pinning. Still wider devices containing non-superconducting barriers can act as Josephson junctions. Accurate patterning of these devices is needed e.g. to match the critical currents of two junctions in a SQUID.

Unfortunately, YBCO can easily be degraded. Oxygen from the copper-oxygen chains diffuses out easily at elevated temperatures and the material itself reacts quite readily with acids and many other chemicals. Wet etching of YBCO in $\mathrm{Na}_{2} \mathrm{H}_{2}$ EDTA has been demonstrated down to less than $20 \mathrm{~nm}$ in $10 \mathrm{~nm}$ thick films [1], but is unreliable in most cases for small patterns in films that are not very thin. Reactive ion etching of YBCO is not yet well developed, mainly due to the difficulty of finding a gas mixture that yields volatile reaction products with yttrium, barium and copper [2],[3]. Ion milling tends to degrade the films very much, unless special precautions (like cooling the sample stage with liquid nitrogen) are taken. The ceramic nature of YBCO usually gives very poor mask selectivity.

In recent work we showed $200 \mathrm{~nm}$ wide lines that were superconducting [4]. These lines were made in an Electron Cyclotron Resonance (ECR) etcher by argon sputter etching with

Manuscript received October 17, 1994.

A. van der Harg, e-mail vanderHarg@DIMES.TUDelft.NL; E. van der Drift, e-mail vanderDrift @DIMES.TUDelft.NL, phone +31-15-786009; P. Hadley, e-mail phadley@sg.tn.tudelft.nl

A.J.M. van der Harg is supported by the Dutch Foundation for Fundamental Research on Matter (FOM) water cooling. The process used then suffered from mask erosion and excessive redeposition. To overcome these problems we developed a thinner mask system containing titanium as the etch resistant layer. Using this mask, lines with widths down to $100 \mathrm{~nm}$ and lengths of $25 \mu \mathrm{m}$ were made. The best $25 \mu \mathrm{m}$ long, $100 \mathrm{~nm}$ wide line obtained thus far has a $\mathrm{T}_{c 0}$ of $84 \mathrm{~K}$.

\section{Sample Preparation}

In our work we mostly use $50 \mathrm{~nm}$ thick films of YBCO deposited onto $\mathrm{SrTiO}_{3}$ by laser ablation. The samples are first cleaned in acetone and 2-propanol. Since we usually work with in-situ deposited gold contacts, the contact pattern is defined next, by standard photolithography and a $\mathrm{KI} / \mathrm{I}_{2}$ etch. For the device etch, the bottom resist layer is $275 \mathrm{~nm}$ of HPR 6512. This layer is baked at $120^{\circ} \mathrm{C}$ for half an hour. Next, $50 \mathrm{~nm}$ of titanium is sputtered on. Low power is used to avoid stress in the growing film that might cause it to buckle. Finally, $90 \mathrm{~nm}$ of Toyo Soda SNR is applied, a negative e-beam resist. The baking recipe for this layer is $60^{\circ} \mathrm{C}$ for 30 minutes.

The resist is exposed in a Leica EBPG-5 with $100 \mathrm{keV}$ electrons. The pattern is aligned to the contacts by markers defined together with the contacts. The resolution of SNR is about $80 \mathrm{~nm}$, but to obtain features of this size special care must be taken in pattern design. The proximity effect in e-beam exposure must be taken into account. Where necessary, patterns are corrected for dose distribution. The SNR is developed in xylene and stopped in 2-propanol. Pattern transfer to the titanium layer takes place by Reactive Ion Etching in a plasma of $15 \mathrm{sccm} \mathrm{Cl} 2,15 \mathrm{sccm} \mathrm{SiCl} 4$ and $17.5 \mathrm{sccm} \mathrm{He}$, process pressure $10 \mu \mathrm{Bar}$ and power density $0.1 \mathrm{~W} / \mathrm{cm}^{2}$.

The samples are now affixed to a 4" silicon wafer with silver paste. (This step is necessary since our ECR etcher accepts only 4" wafers.) The wafer is clamped onto a water cooled, RF driven chuck. Heat transfer is improved by helium back streaming. The actual etching of the YBCO is performed in an Alcatel RCE200 using a plasma of 3 parts argon and 1 part oxygen at a pressure of $1.5 \mu \mathrm{bar}$ and a DC bias of $300 \mathrm{~V}$. Plasma generation is by distributed ECR, independent of substrate biasing. The bottom resist layer is quickly etched anisotropically and the YBCO follows at a rate of $30 \mathrm{~nm} / \mathrm{min}$.

The samples are removed from the wafer and soaked in acetone. A short ultrasound treatment in acetone removes the mask. To dispose of the last remnants of resist, especially on the contacts, the sample is stripped in an oxygen plasma strip- 
per for 10 minutes. The final step is wire bonding of the samples.

\section{Results}

\section{A. Lines}

As a test of the capabilities of the mask and the etch process, we etched long $(25 \mu \mathrm{m})$ lines in a $50 \mathrm{~nm}$ thick YBCO film. The linewidths range from $100 \mathrm{~nm}$ to $1 \mu \mathrm{m}$ in steps of $100 \mathrm{~nm}$. Usually, down to $400 \mathrm{~nm}$ linewidth there is no evidence of any deterioration compared to larger structures. Below $400 \mathrm{~nm}$, a tail appears in the transition. For $100 \mathrm{~nm}$ lines, this tail typically extends some 8-9 $\mathrm{K}$ down.

One of the more remarkable features of this tail is that it does not merge smoothly with the larger transition, but leaves at a sharp angle (see Fig. 1). It is not likely that the tail is due to a lower $T_{c}$ for the line as a whole, since that would imply that the large initial drop were due to the contact pattern. However, given the layout of our devices, the resistance of the $100 \mathrm{~nm}$ line would be 10 times as high as that of the contact pattem, even in the case of equal resistivities. It is really the transition of the line that is measured. The measurements thus show that most of the line has a $T_{c}$ not very different from that of largerscale structures. Apparently there is a small number of weak segments in the line. Even so, we have once measured a $100 \mathrm{~nm}$ wide line that had a $\mathrm{T}_{c 0}$ of $81 \mathrm{~K}$ and a critical current density of $15 \mathrm{MA} / \mathrm{cm}^{2}$ at $75 \mathrm{~K}$.

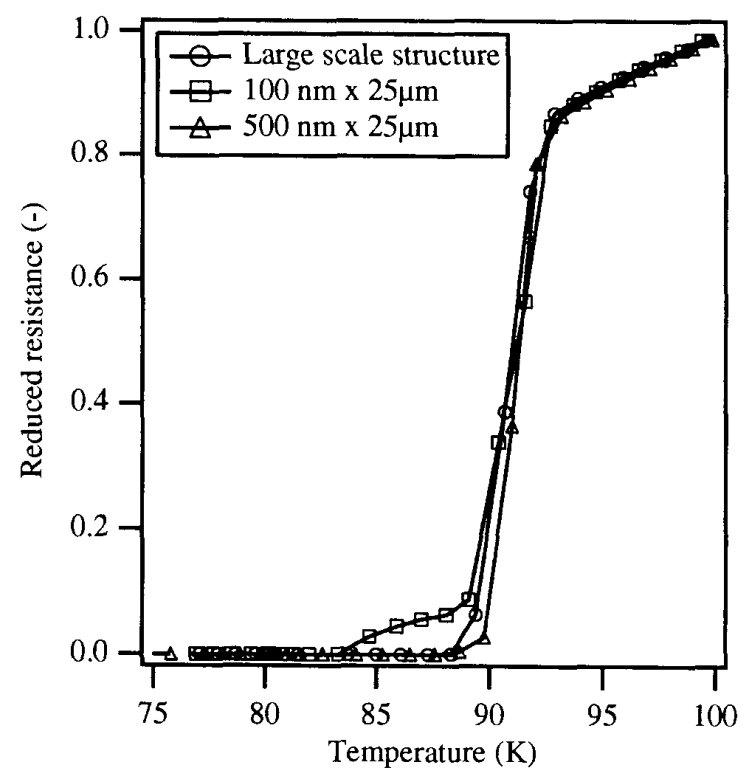

Fig. 1. The resistance of etched lines in a $150 \mathrm{~nm}$ thick film of YBCO, normalized to the resistance at $100 \mathrm{~K}$, as a function of temperature. The length of the line is $25 \mu \mathrm{m}$. The tail in the transition is typical for very narrow lines.

\section{B. Constrictions}

Having proved the feasibility of making extremely narrow structures with good superconducting properties, the next logical step is to fabricate useful devices. Various groups have reported making constrictions which act as Josephson devices [1],[5]. All of the groups that used argon sputtering used liquid nitrogen to cool the sample stage so as to prevent oxygen loss. They also invariably used a positive resist.

Using a positive resist is necessary when the exposure area of the e-beam tool (usually a SEM) is not large enough to expose a complete device. It is then necessary to etch the large-scale structures with a photolithographic mask. The constriction is defined in a separate step, as a small gap in a line. It should be possible to define bridges of around $30 \mathrm{~nm}$ wide and $50-60 \mathrm{~nm}$ long in PMMA. One of the disadvantages of PMMA is the high baking temperature $\left(175^{\circ} \mathrm{C}\right)$ that is needed to obtain the highest resolution. This bake degrades the YBCO. When equipment like an EBPG is available, that can move the substrate in a very controlled way, it would be simpler to expose the entire device in one step. A negative resist then has the advantage of a smaller exposed area and a lower baking temperature $\left(60^{\circ} \mathrm{C}\right.$ for $\mathrm{SNR}$ ). A drawback is the lower resolution, so that the smallest bridges that can be defined have a width of about $100 \mathrm{~nm}$ and a length of approximately 150 to $200 \mathrm{~nm}$.

We made very narrow bridges in $\mathrm{YBCO}$, with a width of down to $100 \mathrm{~nm}$. The superconducting transition of these devices was identical to that of the larger-scale structures. We have measured one sample where a blow-up occurred during the measurement. The critical current at $20 \mathrm{~K}$ dropped by a factor of 100 and the device showed a tail in the transition going

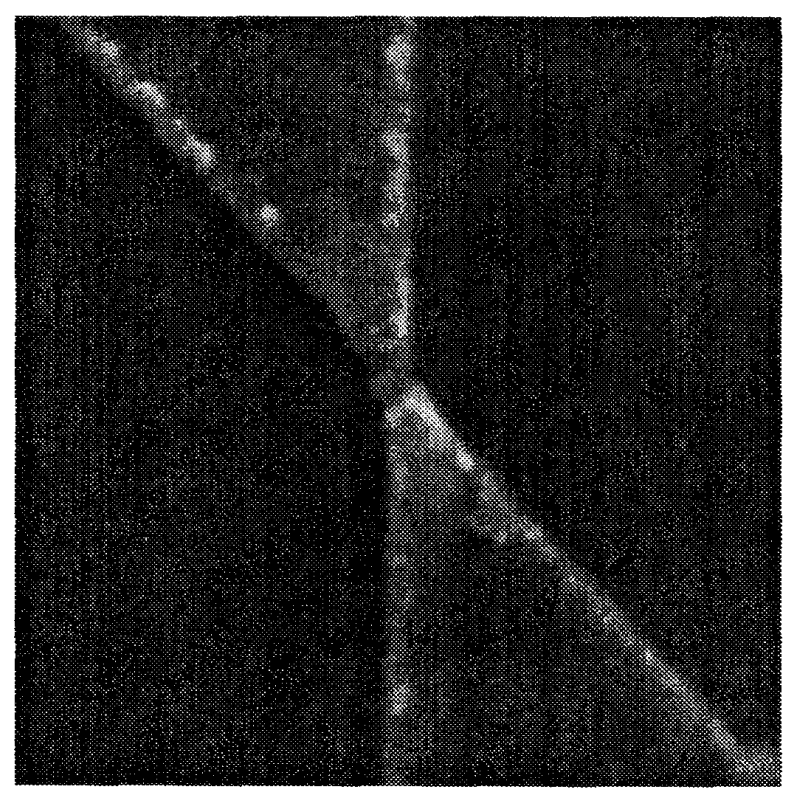

Fig. 2. Top view of a single junction etched into YBCO. The width at the center of the junction is $147 \mathrm{~nm}$. 
down to about $30 \mathrm{~K}$. A possible explanation for this behavior is that the heating that accompanies the breakdown allows oxygen to escape from the bridge region. The small width of the bridge (less than $200 \mathrm{~nm}$ ) would make this a very rapid process.

Since it is obvious in this case that the structure is severely damaged it is not surprising that the superconducting properties are influenced to such a large extent. The occurrence of a smaller tail in longer lines might indicate a more limited oxygen loss in parts of the line, reaching across the full width of the line in a limited number of places. Given the two-plateau dependence of $\mathrm{T}_{c}$ on oxygen content and the quite steep curve between the plateaus, the degraded places might be just off the edge of the $90 \mathrm{~K}$ plateau.

The superconducting bridges we have manufactured so far have very high critical current densities, up to tens of $\mathrm{MA} / \mathrm{cm}^{2}$ at $50 \mathrm{~K}$. This shows that our fabrication process does not damage the structures on a scale of 50 to $100 \mathrm{~nm}$.

A drawback is that the microwave power needed to induce steps in the I-V curve is quite high. The steps result from the coupling of vortex motion to the applied RF field. On the first step exactly one vortex-antivortex pair crosses the bridge per oscillation, on the second two and so on. The process is similar to Josephson tunneling where the applied RF-current induces tunneling of Cooper pairs across a barrier. In fact, the relation between voltage and frequency is exactly the same [5]. We have not been able to produce these steps. We believe that we cannot couple enough power into the junction. Extrapolating from the results of de Nivelle [5], who needed at least $-10 \mathrm{dBm}$ at $10 \mathrm{GHz}$ to induce Shapiro steps in a junction with a critical current of $0.3 \mathrm{~mA}$ at $55 \mathrm{~K}$, we would need to couple more than $16 \mathrm{dBm}$ into one of our typical junctions with a critical current of $6 \mathrm{~mA}$ to induce barely visible steps the $\mathrm{I}-\mathrm{V}$ curve.

\section{Constriction Arrays}

Single junctions are interesting for the occurrence of periodic vortex motion and their locking on an externally applied high frequency field. Josephson junction arrays are also interesting for their reaction to these fields. It is well known from low- $T_{c}$ work that entire arrays may lock phase, giving rise to so-called "giant Shapiro steps" [6],[7]. Depending on the orientation of the array with respect to the applied current, fractional vortex filling of the array can also give rise to steps, leading to fractional giant Shapiro steps. It would be very interesting to see if something similar happens in High- $\mathrm{T}_{c}$ constriction arrays. An added benefit of using High- $\mathrm{T}_{c}$ materials is that the size of the superconducting islands can be varied from far above to far below the effective magnetic penetration depth. This should have its effect on the phase locking of the array.

The same process that we use for making single bridges can also be used very well for making arrays. Fig. 3 shows a top view of such an array. It shows that the constrictions are all in place and that their size is reasonably uniform. The same measurement problem that plagues us in single bridges is even more manifest here. To induce steps in an array would require very high power levels, which we don't have available in our setup.

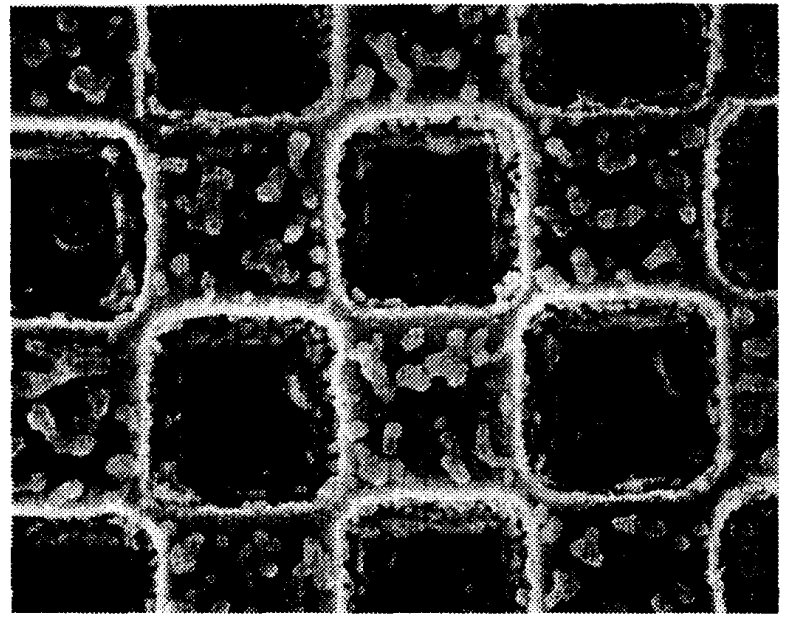

Fig. 3. Top view of a processed constriction array. The debris on top is the result of a very short ultrasonic treatment to remove some of the redeposition. The titanium mask is still in place. The individual islands are $500 \mathrm{~nm}$ across.

An additional problem is that any small resistance in the current path causes a large voltage drop with the large currents that we need to drive array samples normal. Most precision current sources cannot handle this situation.

\section{SUMMARY AND CONCLUSIONS}

We have succeeded in making extremely long and narrow lines in $\mathrm{YBa}_{2} \mathrm{Cu}_{3} \mathrm{O}_{7}$. We have developed a mask that can be removed without exposing the vulnerable lines to water or reactive plasmas. The resulting lines have high critical temperatures and high critical current densities. Measurements on constrictions show that they often have very high critical currents.

In order to make these constrictions useful, it will be necessary to make them even narrower or to reduce the film thickness in the constriction. In their present state the amount of power necessary to drive the junctions is too high. This is even more true for constriction arrays.

Our results indicate that it is not necessary to use liquid nitrogen cooling to achieve good-quality samples. As long as the sample is not allowed to heat up to much more than the highest temperature that is used in the mask process and the etch time is kept short, oxygen diffusion is minor. The prerequisites for this are good heat conduction between the sample and a cooled sample stage, and a high etch rate.

\section{REFERENCES}

[1] J.R.Wendt et al.," $\mathrm{YBa}_{2} \mathrm{Cu}_{3} \mathrm{O}_{7}$ nanobridges fabricated by direct-write electron beam lithography," Appl. Phys. Lett., vol. 61, pp. 1597-1599, 28 September 1992

[2] M. G. Heijman, "Fine structuring of magnetic and high- $T_{c}$ superconducting ceramic oxides in an $\mathrm{HCl}$ plasma," Appl. Surf. Sci., vol. 36, pp. 362-372, 1989.

[3] T. Oishi, T. Takami, K. Kuroda, K. Kojima, O. Wada and M. Nunoshita, "Reactive ion etching of $\mathrm{BiSrCaCuO}$ Superconducting thin films using 
ethane and oxygen," Jpn. J. Appl. Phys., vol. 33, pp. L315-L317, March 1994.

[4] H. Assink et al.,"Critical currents in submicron $\mathrm{YBa}_{2} \mathrm{Cu}_{3} \mathrm{O}_{7}$ lines," IEEE Trans. Appl. Supercond., vol. 3, pp. 2983-2985, March 1993.

[5] Martin de Nivelle, "Transport properties of nanobridges in high- $\mathrm{T}_{c}$ superconducting $\mathrm{YBa}_{2} \mathrm{Cu}_{3} \mathrm{O}_{7}$ films," Ph.D. thesis, Twente University, 1993.
[6] Dick Eikmans, "Critical and dynamical behavior of Josephson-junction arrays," Ph. D. thesis, Institute for Theoretical Physics, State University Utrecht, 1991

[7] Lydia Lee Sohn,"Geometrical effects in two-dimensional arrays of Josephson junctions," Ph. D. thesis, Harvard University, 1992 